\title{
Comparing the Safety and Efficacy of Celecoxib for the Treatment of Osteoarthritis in Asian and non- Asian Populations: An Analysis of Data from Two Randomized, Double-blind, Placebo-controlled, Active-comparator Trials
}

\author{
Andri Lubis · Wei Wang · Graça Lima • Rana Fayyad • \\ Chris Walker
}

Received: May 22, 2017 / Published online: September 18, 2017

(C) The Author(s) 2017. This article is an open access publication

\section{ABSTRACT}

Introduction: Celecoxib is an effective treatment for pain associated with osteoarthritis. There are differences in patient demographics among ethnic groups, with Asian populations typically smaller in body size. As a consequence, there may be a perception that celecoxib is less effective, or has poorer tolerability in Asian patients.

Methods: This analysis compares data from two multicenter, randomized, double-blind, placebo-controlled, active-comparator trials of celecoxib for the treatment of osteoarthritis of the knee: one study in Asian patients and the

Enhanced content To view enhanced content for this article go to.http://www.medengine.com/Redeem/ 480CF0606BAE528C.

\section{A. Lubis}

Faculty of Medicine, Universitas Indonesia/Cipto

Mangunkusumo Hospital, Jakarta, Indonesia

W. Wang

Orthpedic Department, Peking Union Medical

College Hospital, Beijing, China

G. Lima

Pfizer Inc, Quarry Bay, Hong Kong

R. Fayyad

Pfizer Inc, New York, NY, USA

C. Walker $(\square)$

Pfizer Inc, Walton Oaks, Surrey, UK

e-mail: chris.walker@pfizer.com other in a mixed population comprised mostly of non-Asian patients (from which Asian patients were excluded for this analysis). Each trial was of similar design, with patients randomized 2:2:1 to 6 weeks treatment with celecoxib $200 \mathrm{mg}$ once daily, active comparator (naproxen $500 \mathrm{mg}$ twice daily or ibuprofen $800 \mathrm{mg}$ three times daily), or placebo. The primary efficacy endpoint in each trial was the change from baseline to week 6 in the Patient's Assessment of Arthritis Pain, as measured on a visual analog scale.

Results: In total, 329 patients were included in the efficacy analysis, 179 in the Asian study and 150 in the non-Asian study. The Asian population was significantly older and smaller in body size $(P<0.0001)$. There was no significant difference between the Asian and non-Asian populations in change in pain score $(95 \%$ confidence interval) at study endpoint with celecoxib $[-1.1(-7.7,5.5) ; P=0.7400]$ or placebo $[-5.2(-14.8,4.4) ; P=0.2870]$. There were also no notable differences in safety outcomes between populations.

Conclusions: Due to the smaller size of some Asian patients with OA, physicians may be tempted to decrease the dose of celecoxib below the therapeutic range recognized by regulatory authorities; these data suggest that dose changes are not necessary.

Funding: Pfizer Inc. 
Keywords: Asian; Celecoxib; Osteoarthritis; Pain; Race

\section{INTRODUCTION}

The efficacy of celecoxib for the treatment of osteoarthritis (OA) has been well established, both in largely Caucasian populations [1-3] and in patients of various other ethnicities, including Asian patients [4-6]. The efficacy of other nonsteroidal anti-inflammatory drugs (NSAIDs) for the treatment of $\mathrm{OA}$ has also been well established in a number of trials conducted in Asian countries $[7,8]$.

There are commonly differences in body mass and body shape between Asian patients and patients of other ethnicities, with Asian patients often being smaller and having a lower body mass index [9]. As a result, there is a perception that Asian patients may require lower doses of medications, including treatments for pain [8], and there is an unsupported assumption by some healthcare professionals that celecoxib treatment may be less efficacious, or should be utilized at lower doses, in Asian patients with OA.

This report is a comparison of the results from two randomized clinical trials of celecoxib for the treatment of OA: one in Asian patients and one in non-Asian patients. Direct comparison of the efficacy and safety of celecoxib in these two populations aims to assess the possibility of any significant difference in response to, or tolerance of, OA treatment in Asian patients.

\section{METHODS}

\section{Study Populations}

This article is based on previously conducted studies, and does not involve any new studies of human or animal subjects performed by any of the authors. Study A3191052 ("Asian" study) was a 6-week, randomized, double-blind, placebo-controlled study to determine the efficacy and safety of once-daily celecoxib versus twice-daily naproxen in Asian patients with OA of the knee in the United States [5]. Patients were randomized 2:2:1 to celecoxib $200 \mathrm{mg}$ once daily, naproxen $500 \mathrm{mg}$ twice daily, or placebo. The study is registered on ClinicalTrials.gov (NCT00620867).

To select the most appropriate comparator trial, all Pfizer-sponsored studies of celecoxib for the treatment of OA were reviewed based on the following criteria: only randomized, controlled trials of celecoxib of comparable duration; conducted in a mixed population in which the majority of patients were non-Asian; included an active comparator that is currently available to patients; and included efficacy endpoints that were comparable to study A3191052. Ten Pfizer-sponsored studies of celecoxib for the treatment of OA were identified, of which only one study met all of the above criteria. Study A3191063 ("non-Asian" study) was a 6-week, multicenter, randomized, double-blind, placebo-controlled, active-comparator, parallel-group study in patients with OA of the knee. Patients were randomized 2:2:1 to celecoxib $200 \mathrm{mg}$ once daily, ibuprofen $800 \mathrm{mg}$ three times daily, or placebo. The study was conducted in 32 centers in the United States in a mixed population of which $89.6 \%$ were nonAsian. Asian patients were excluded for this analysis. Study A3191052 is not registered on ClinicalTrials.gov.

Both trials were conducted between 2002 and 2003. Patients were included in either study if they were diagnosed with OA of the knee according to the American College of Rheumatology (ACR) guidelines [10], had a functional capacity class of I-III, were eligible, in the investigator's opinion, for daily therapy with an NSAID, and had completed a washout period of $\geq 2$ days prior to baseline assessments. Patients taking $\leq 325 \mathrm{mg}$ acetylsalicylic acid daily for cardiovascular prophylaxis and who had been on a stable dose for 30 days were also included.

In the Asian study, patients were aged $\geq 45$ years and of self-reported Asian descent. In the non-Asian study, patients were aged $\geq 40$ years. Patients were excluded from either study if they had: inflammatory arthritis, acute joint trauma, active GI disease, been diagnosed with unstable cardiovascular disease within the past 6 months, or had taken acetaminophen 
within $24 \mathrm{~h}$ of the baseline visit (acetaminophen was permitted as rescue medication).

In both studies, the primary efficacy outcome was the change from baseline to week 6 in the Patient's Assessment of Arthritis Pain, as measured on a visual analog scale (VAS; measured from $0-100 \mathrm{~mm}$ ). As a secondary outcome, both trials assessed the Western Ontario and McMaster Universities Osteoarthritis (WOMAC) Index, a self-administered questionnaire consisting of 24 items: five regarding pain, two regarding stiffness, and 17 regarding physical function. Responses to each question ranged from " 0 " (none) to " 4 " (extreme). The total score (ranging from 0 to 96 ) is reported with a negative change from baseline, indicating improvement. Safety was assessed by measuring adverse events (AEs) and upper gastrointestinal (UGI) tolerability. A patient was determined to have had a UGI event if they reported at least one of the following: moderate or severe nausea, moderate or severe abdominal pain, or moderate or severe dyspepsia.

Data on patient demographics, change in VAS and WOMAC, and safety were compared for each trial. All Asian patients in the nonAsian trial (10.4\% of all patients, $8.4 \%$ of whom were treated with celecoxib or placebo) were excluded from the analysis. For patient demographics, $P$ values were determined by $t$ test or by Chi-square test. For efficacy assessments, least squares (LS) means and $P$ values are from the treatment-study interaction, based on an analysis of variance model, with change from baseline as the dependent variable; study, baseline, and treatment as the significant demographics in the comparison; and treatment-study interaction as the covariates. The primary efficacy analysis was conducted on the efficacy-evaluable population, which included patients who were randomized, received at least one dose of study medication, and who had no major protocol violations; both baseline and Week 6 assessments of the primary efficacy variable; adequate treatment (i.e., compliance rate of $70-120 \%$ during the 6 -week study period); and had taken study medication within $48 \mathrm{~h}$ prior to week 6 visit (because the minimum washout period of ibuprofen was 2 days). Safety outcomes are reported for Asian and non-Asian patients who were randomized and received at least one dose of study medication.

\section{RESULTS}

This analysis included a total of 441 patients randomized to celecoxib or placebo; of these, 329 efficacy-evaluable patients were included in the primary efficacy analysis. The Asian population was significantly older and smaller in body size, and had a shorter duration of OA than those in the non-Asian population $(P<0.005$ for each, Table 1$)$. In the Asian population, 121 patients were treated with celecoxib and 58 treated with placebo. In the non-Asian population, 108 patients were treated with celecoxib and 42 with placebo. A comparison of the change in pain score with celecoxib and placebo in each trial indicated no significant difference between Asian and nonAsian patients (Table 2).

In the Asian population, mean (standard error [SE]) WOMAC Total Domain Scores at baseline were 50.5 (1.3) and 50.2 (1.9), with an LS mean (SE) change at endpoint of -29.0 (1.8) and -23.7 (2.4), for celecoxib and placebo, respectively. In the non-Asian population, mean (SE) WOMAC scores were 52.6 (1.0) and 57.6 (1.8) at baseline, and the change at endpoint (SE) was -24.7 (1.6) and -19.6 (2.5), for celecoxib and placebo, respectively. There was no significant difference between the two populations in the change at week 6 with celecoxib $(P=0.0842)$ or placebo $(P=0.2475)$.

Treatment-emergent all-causality AEs were broadly similar in the Asian and non-Asian populations (Table 3). The percentage of patients with AEs with celecoxib were comparable in each population, wherein the most common AEs were abdominal pain, depression, and headache. The percentage of all patients with AEs was lower in those treated with celecoxib than with the active comparator: $(27.6 \%$ of patients with celecoxib versus $46.1 \%$ with naproxen in the Asian trial; $31.9 \%$ with celecoxib versus $38.3 \%$ with ibuprofen in the non-Asian trial). There were fewer UGI events with celecoxib than with the active comparator 
Table 1 Patient demographics in Asian and non-Asian trial populations

\begin{tabular}{|c|c|c|c|c|c|}
\hline & \multicolumn{2}{|l|}{$\operatorname{Asian}^{\mathrm{a}}$} & \multicolumn{2}{|l|}{ Non-Asian ${ }^{b}$} & \multirow[t]{2}{*}{$P$ value } \\
\hline & Celecoxib & Placebo & Celecoxib & Placebo & \\
\hline$n$ & 145 & 78 & 152 & 66 & \\
\hline Gender, $n(\%)$ & & & & & $0.1177^{\mathrm{c}}$ \\
\hline Female & $97(67)$ & $52(67)$ & $88(58)$ & $42(64)$ & \\
\hline Male & $48(33)$ & $26(33)$ & $64(42)$ & $24(36)$ & \\
\hline Age, mean (SD), years & $65.9(11.1)$ & $63.9(11.1)$ & $58.4(10.9)$ & $55.6(10.4)$ & $<0.0001^{\mathrm{d}}$ \\
\hline Race, $n(\%)$ & & & & & $<0.0001^{\mathrm{c}}$ \\
\hline Asian & $145(100)$ & $78(100)$ & 0 & 0 & \\
\hline White & 0 & 0 & $93(61)$ & $39(59)$ & \\
\hline Black & 0 & 0 & $37(24)$ & $16(24)$ & \\
\hline Other & 0 & 0 & $22(14)$ & $11(17)$ & \\
\hline Weight, mean (SD), kg & $66.5(13.3)$ & $65.6(12.4)$ & $87.9(19.3)$ & $89.0(22.2)$ & $<0.0001^{\mathrm{d}}$ \\
\hline Height, mean (SD), cm & $158.6(8.0)$ & $158.2(7.5)$ & $168.9(9.7)$ & $165.8(10.7)$ & $<0.0001^{\mathrm{d}}$ \\
\hline BMI, mean $(\mathrm{SD}), \mathrm{kg} / \mathrm{m}^{2}$ & $26.4(4.8)$ & $26.1(4.2)$ & $30.7(5.8)$ & $32.4(7.5)$ & $<0.0001^{\mathrm{d}}$ \\
\hline \multicolumn{6}{|l|}{ Duration of OA, years } \\
\hline Mean (SD) & $4.5(4.0)$ & $4.6(4.3)$ & $6.5(7.4)$ & $6.2(8.1)$ & $0.0021^{\mathrm{d}}$ \\
\hline Median & 3.0 & 3.2 & 3.1 & 4.0 & \\
\hline
\end{tabular}

$B M I$ body mass index, $O A$ osteoarthritis, $S D$ standard deviation

a Patients from study A3191052

b Patients from study A3191063, excluding Asian patients

c Assessed by Chi-square test

d Assessed by $t$ test

in both full trial populations $(3.4 \%$ of patients with celecoxib versus $6.4 \%$ with naproxen in the Asian trial; $1.9 \%$ with celecoxib versus $5.8 \%$ with ibuprofen in the non-Asian trial.

\section{DISCUSSION}

In this comparison of the efficacy and safety of celecoxib for the treatment of OA of the knee, there was no significant difference between a population of Asian patients and a mixed population of non-Asian patients. This comparison benefits from utilizing patient-level data from two trials of notably similar design. Both trials were conducted in the United States over a similar time period, employed the same inclusion and exclusion criteria, and assessed the same efficacy endpoints.

It is notable that despite significant clinical and demographic differences between the Asian and non-Asian populations in this analysis, with Asian patients being significantly shorter, having lower body weight, being older, and having a shorter duration of disease than non-Asian patients (which may have led to differences in treatment pharmacokinetics and pharmacodynamics), efficacy and safety outcomes in each trial were similar. The finding that, in addition to pain assessments, there was 
Table 2 Change in pain score (VAS) at endpoint in Asian and non-Asian trial populations

\begin{tabular}{|c|c|c|c|c|}
\hline & \multicolumn{2}{|l|}{$\operatorname{Asian}^{a}$} & \multicolumn{2}{|l|}{ Non-Asian ${ }^{b}$} \\
\hline & Celecoxib & Placebo & Celecoxib & Placebo \\
\hline$n$ & 121 & 58 & 108 & 42 \\
\hline Baseline, mean (SE) & $65.1(1.1)$ & $63.7(1.5)$ & $68.4(1.1)$ & $66.2(2.0)$ \\
\hline Week 6, mean (SE) & $21.7(1.9)$ & $25.6(3.1)$ & $28.5(2.2)$ & $34.9(3.8)$ \\
\hline Change from baseline, LS mean (SE) & $-41.4(2.3)$ & $-38.6(3.2)$ & $-40.3(2.3)$ & $-33.4(3.5)$ \\
\hline \multicolumn{5}{|l|}{ Difference in LS mean vs. non-Asian ${ }^{c}$} \\
\hline LS mean & -1.1 & -5.2 & - & - \\
\hline $95 \%$ confidence interval & $-7.7,5.5$ & $-14.8,4.4$ & - & - \\
\hline$P$ value & 0.7400 & 0.2870 & - & - \\
\hline
\end{tabular}

$L S$ least squares, $S E$ standard error, $V A S$ visual analog scale

${ }^{a}$ Patients from study A3191052

b Patients from study A3191063, excluding Asian patients

${ }^{c}$ LS mean and $P$ value are for treatment-study interaction, based on a general linear model with change from baseline as the dependent variable; with study, baseline, and treatment as the significant demographics in the comparison; and treatment-study interaction as the covariant

also no significant difference in the improvement in patient function, as assessed by WOMAC score, supports this conclusion. There appeared to be a trend toward a greater change in pain score with placebo in the Asian population; however, this was not significantly different from the placebo response in the non-Asian population.

Although safety outcomes in each population were similar, discontinuations due to AEs were numerically greater in the Asian population, as was the incidence of abdominal pain. The total proportion of patients who discontinued was similar for patients in the Asian and non-Asian trials with celecoxib $(18.6 \%$ vs. $18.2 \%$, respectively) and placebo $(24.4 \%$ vs. $26.0 \%$, respectively). The reasons for discontinuation were also similar in each trial, with protocol violation and patient default being the most common causes. These studies were not designed, or powered, to evaluate cardiovascular safety; however, all NSAIDs have been associated with an increased risk of myocardial infarction with no notable difference between celecoxib and traditional NSAIDs [11, 12].

\section{LIMITATIONS}

Responses to some treatments have been suggested to vary across ethnic groups, potentially based on a degree of "ethnic sensitivity" in the pharmacokinetics and pharmacodynamics of some treatments [13]. While not the focus of the design of these studies or a specific aim of this analysis, there have been shown to be differences in the perception of pain in individual patients with rheumatic disease, with factors such as depression and catastrophizing being associated with reported severity of pain, sensitivity to pain, disability, and poor treatment outcomes [14]. Some studies have demonstrated differences between ethnic groups in the perception of pain intensity [15-19]. However, others have suggested that, when confounding variables are accounted for, any ethnic differences in perception of pain intensity are minimal [20]. The latter conclusion is consistent with the findings of this analysis, wherein Asian patients with OA did not differ from non-Asian patients in their response to celecoxib. However, this conclusion is limited by the fact that 
Table 3 Adverse events (all-causality) in Asian and non-Asian trial populations

\begin{tabular}{|c|c|c|c|c|}
\hline & \multicolumn{2}{|l|}{$\operatorname{Asian}^{\mathrm{a}}$} & \multicolumn{2}{|c|}{ Non-Asian ${ }^{b}$} \\
\hline & Celecoxib & Placebo & Celecoxib & Placebo \\
\hline$n$ & 145 & 76 & 147 & 65 \\
\hline Number of AEs & 64 & 33 & 81 & 44 \\
\hline Patients with AEs, $n(\%)$ & $40(27.6)$ & $22(28.9)$ & $49(33.3)$ & $26(40.0)$ \\
\hline Patients with serious AEs, $n$ (\%) & 0 & 0 & $1(0.7)$ & 0 \\
\hline Patients discontinued due to AEs, $n$ (\%) & $10(6.9)$ & $3(3.9)$ & $5(3.4)$ & $5(7.7)$ \\
\hline \multicolumn{5}{|l|}{ GI-related AEs, $n(\%)$} \\
\hline Abdominal pain & $11(7.6)$ & $3(3.9)$ & $4(2.7)$ & $3(4.6)$ \\
\hline Dyspepsia & $2(1.4)$ & 0 & $4(2.7)$ & $1(1.5)$ \\
\hline Constipation & 0 & 0 & $1(0.7)$ & $1(1.5)$ \\
\hline Diarrhea & $2(1.4)$ & 0 & $1(0.7)$ & 0 \\
\hline Nausea & $1(0.7)$ & $1(1.3)$ & $6(4.1)$ & 0 \\
\hline \multicolumn{5}{|l|}{ CNS-related AEs, $n(\%)$} \\
\hline Depression & $11(7.6)$ & $11(14.5)$ & $12(8.2)$ & $9(13.8)$ \\
\hline Dizziness & 0 & $1(1.3)$ & 0 & $1(1.5)$ \\
\hline Headache & $3(2.1)$ & 0 & $6(4.1)$ & $3(4.6)$ \\
\hline
\end{tabular}

$A E$ adverse event, $C N S$ central nervous system, $G I$ gastrointestinal

a Study A3191052 conducted in Asian patients

b Study A3191063, excluding Asian patients

these studies were not designed to assess ethnic differences in perception of pain intensity and, as such, do not provide any specific new information to address this question. At the same time, it should be noted that the Asian population in this analysis would likely consist of multiple ethnic groups from throughout Asia. Although the patients in this analysis were not genetically tested, there could be significant differences between Asian patients and nonAsian patients in the activity of cytochrome P450 2C9 (CYP2C9), the principal metabolizer of celecoxib [21], which could confound the interpretation of these data.

That the Asian population in this comparison was based in the United States could also be perceived as a limitation, as there will be cultural differences between this population and patients living in Asia. It has been suggested that differences in pain perception in Asian populations may actually be driven by acculturation, with patients who are in the process of adapting, culturally and physiologically, to a new culture experiencing heightened pain responses, although this only affects the first-generation of relocated individuals [22]. If this is the case, then this comparison, with an Asian population based in the United States, may be more likely to detect differences in the response to celecoxib.

\section{CONCLUSIONS}

This analysis did not detect any significant difference between Asian and non-Asian patients in the response to celecoxib for treatment of OA of the knee despite differences in size, age, and 
duration of disease. This information will likely be useful for physicians assessing treatment options for Asian patients with OA. Due to the smaller size of some Asian patients with OA, physicians may be tempted to decrease the dose of celecoxib below the therapeutic range recognized by regulatory authorities; these data suggest that dose changes are not necessary.

\section{ACKNOWLEDGEMENTS}

This study and the article processing charges were funded by Pfizer Inc. Medical writing support was provided by Joshua Fink, PhD, of Engage Scientific Solutions, and was funded by Pfizer. All authors had full access to all of the data in this study and take complete responsibility for the integrity of the data and accuracy of the data analysis. All named authors meet the International Committee of Medical Journal Editors (ICMJE) criteria for authorship for this manuscript, take responsibility for the integrity of the work as a whole, and have given final approval to the version to be published.

Disclosures. Andri Lubis has nothing to disclose. Wei Wang has received consultancy fees from Pfizer, Bayer, Novartis, GSK, Roche, Sanofi-Aventis, Johnson \& Johnson, Stryker, and Zimmer. Graça Lima is an employee of Pfizer and holds stock options with Pfizer. Rana Fayyad is an employee of Pfizer and holds stock options with Pfizer. Chris Walker is an employee of Pfizer and holds stock options with Pfizer.

Compliance with Ethics Guidelines. This article is based on previously conducted studies, and does not involve any new studies of human or animal subjects performed by any of the authors.

Data Availability. The datasets analyzed during the current study are available from the corresponding author on reasonable request.
Open Access. This article is distributed under the terms of the Creative Commons Attribution-NonCommercial 4.0 International License (http://creativecommons.org/licenses/ by-nc/4.0/), which permits any noncommercial use, distribution, and reproduction in any medium, provided you give appropriate credit to the original author(s) and the source, provide a link to the Creative Commons license, and indicate if changes were made.

\section{REFERENCES}

1. Bensen WG, Fiechtner JJ, McMillen JI, et al. Treatment of osteoarthritis with celecoxib, a cyclooxygenase-2 inhibitor: a randomized controlled trial. Mayo Clin Proc. 1999;74:1095-105.

2. Kivitz AJ, Moskowitz RW, Woods E, et al. Comparative efficacy and safety of celecoxib and naproxen in the treatment of osteoarthritis of the hip. J Int Med Res. 2001;29:467-79.

3. Williams GW, Ettlinger RE, Ruderman EM, et al. Treatment of osteoarthritis with a once-daily dosing regimen of celecoxib: a randomized, controlled trial. J Clin Rheumatol. 2000;6:65-74.

4. Essex MN, O'Connell M, Bhadra Brown P. Response to nonsteroidal anti-inflammatory drugs in African Americans with osteoarthritis of the knee. J Int Med Res. 2012;40:2251-66.

5. Essex MN, O'Connell MA, Behar R, Bao W. Efficacy and safety of nonsteroidal anti-inflammatory drugs in Asian patients with knee osteoarthritis: summary of a randomized, placebo-controlled study. Int J Rheum Dis. 2016;19:262-70.

6. Essex MN, Behar R, O'Connell MA, Brown PB. Efficacy and tolerability of celecoxib and naproxen versus placebo in Hispanic patients with knee osteoarthritis. Int J Gen Med. 2014;7:227-35.

7. Yoo MC, Yoo WH, Kang SB, et al. Etoricoxib in the treatment of Korean patients with osteoarthritis in a double-blind, randomized controlled trial. Curr Med Res Opin. 2014;30:2399-408.

8. Sakamoto C, Soen S. Efficacy and safety of the selective cyclooxygenase- 2 inhibitor celecoxib in the treatment of rheumatoid arthritis and osteoarthritis in Japan. Digestion. 2011;83:108-23. 
9. WHO Expert Consultation. Appropriate body-mass index for Asian populations and its implications for policy and intervention strategies. Lancet. 2004;363:157-63.

10. Altman R, Asch E, Bloch D, et al. Development of criteria for the classification and reporting of osteoarthritis. Classification of osteoarthritis of the knee. Diagnostic and Therapeutic Criteria Committee of the American Rheumatism Association. Arthritis Rheum. 1986;29:1039-49.

11. Bally M, Dendukuri N, Rich B, et al. Risk of acute myocardial infarction with NSAIDs in real world use: Bayesian meta-analysis of individual patient data. BMJ. 2017;357:j1909.

12. Nissen SE, Yeomans ND, Solomon DH, et al. Cardiovascular safety of Celecoxib, Naproxen, or Ibuprofen for Arthritis. N Engl J Med. 2016;375:2519-29.

13. Yasuda SU, Zhang L, Huang SM. The role of ethnicity in variability in response to drugs: focus on clinical pharmacology studies. Clin Pharmacol Ther. 2008;84:417-23.

14. Edwards RR, Cahalan C, Mensing G, Smith M, Haythornthwaite JA. Pain, catastrophizing, and depression in the rheumatic diseases. Nat Rev Rheumatol. 2011;7:216-24.

15. Riley JL 3rd, Wade JB, Myers CD, Sheffield D, Papas RK, Price DD. Racial/ethnic differences in the experience of chronic pain. Pain. 2002;100:291-8.
16. Edwards CL, Fillingim RB, Keefe F. Race, ethnicity and pain. Pain. 2001;94:133-7.

17. Campbell CM, Edwards RR. Ethnic differences in pain and pain management. Pain Manag. 2012;2:219-30.

18. Al-Hashimi M, Scott S, Griffin-Teall N, Thompson J. Influence of ethnicity on the perception and treatment of early post-operative pain. $\mathrm{Br} \mathrm{J}$ Pain. 2015;9:167-72.

19. Rowell LN, Mechlin B, Ji E, Addamo M, Girdler SS. Asians differ from non-Hispanic Whites in experimental pain sensitivity. Eur J Pain. 2011;15:764-71.

20. Edwards RR, Moric M, Husfeldt B, Buvanendran A, Ivankovich $\mathrm{O}$. Ethnic similarities and differences in the chronic pain experience: a comparison of African American, Hispanic, and white patients. Pain Med. 2005;6:88-98.

21. Schwarz UI. Clinical relevance of genetic polymorphisms in the human CYP2C9 gene. Eur J Clin Invest. 2003;33(Suppl 2):23-30.

22. Chan MY, Hamamura T, Janschewitz K. Ethnic differences in physical pain sensitivity: role of acculturation. Pain. 2013;154:119-23. 